\title{
大型线性方程组的迭代求解
}

\author{
陈飞武* 赵小红 \\ (北京科技大学化学系, 北京 100083)
}

\begin{abstract}
摘要: 将求解大型标准特征值问题的 Davidson 方法推广到求解大型线性方程组. 推广后的方法既可以用于求 解对称问题, 又可以用于求解非对称问题. 数值计算结果显示, 该方法对这两类问题的求解分别优于共轭梯度方 法和双共轭梯度方法.
\end{abstract}

关键词： 线性方程组；Davidson 方法；共轭梯度方法；双共轭梯度方法 中图分类号：0641

\section{Iterative Solution of Large-Scale Linear Equations}

\author{
CHEN Fei-Wu* ZHAO Xiao-Hong \\ (Department of Chemistry, University of Science and Technology Beijing, Beijing 100083, P. R. China)
}

\begin{abstract}
The Davidson's method originally developed for eigenvalue problems was extended to solve large-scale linear equations. It can be used for symmetrical and nonsymmetrical matrices. Numerical results presented in this study demonstrate that the present scheme is much more effective than conjugate gradient method and biconjugate gradient method for symmetrical and nonsymmetrical problems, respectively.
\end{abstract}

Key Words: Linear equations; Davidson's method; Conjugate gradient method; Biconjugate gradient method

Linear systems of equations are associated with many problems ${ }^{[1-8]}$ in physics, chemistry, engineering, and geophysics. The typical examples in quantum mechanical calculations are related to problems encountered in the linear coupled Hartree-Fock equations ${ }^{[9]}$ and coupled-cluster method ${ }^{[0]}$, in computing nonlinear optical properties of correlated conjugated systems ${ }^{[11,12]}$, and surface charges of a solvent cavity that nominally encloses the solute in dielectric continuum theory ${ }^{[13]}$.

For small systems there are well-established direct algorithms ${ }^{[-3]}$ such as Gaussian elimination to obtain an accurate solution of a set of linear equations. However, for large dimensional systems iterative approaches ${ }^{[1-3]}$ are usually more efficient in terms of the computer storage and the computational speed. Among them the conjugate gradient method (CGM) $)^{[1-3,14]}$ and biconjugate gradient method (BCGM) ${ }^{[3]}$ are often exploited to find a solution for a symmetrical and positive definite matrix and a nonsysmmetrical matrix, respectively. The generalized minimum residual method ${ }^{[2,15-17]}$ is another general purpose method. Its performance is more or less equivalent to the CGM and BCGM. More iterative techniques could be found from Refs.[1-3].

Davidson's method ${ }^{[1,19]}$ is a very effective approach for a largescale standard eigenvalue problem. Lenthe and Pulay ${ }^{[20]}$ pointed out that expansion vectors in Davidson's method could be truncated to two vectors without significant reduction in the speed of convergence as the dimension of the subspace became large. Davidson's method has been extended to calculate a few lowest eigenvalues and corresponding eigenvectors of large generalized eigenvalue problem ${ }^{[21,22]}$. It is the purpose of this study to derive an analogues version of the Davidson's method for solution of linear equations. Numerical results were presented and compared with the conjugate gradient method for symmetrical systems and also with the biconjugate gradient method for nonsymmetrical systems.

Received: May 29, 2009; Revised: July 16, 2009; Published on Web: August 27, 2009.

*Corresponding author. Email: chenfeiwu@sas.ustb.edu.cn; Tel: +86-10-62332689.

The project was supported by the National Natural Science Foundation of China (20473011, 20773011).

国家自然科学基金(20473011, 20773011)资助项目

C Editorial office of Acta Physico-Chimica Sinica 


\section{Theory}

A set of linear equations has the following matrix form: $\boldsymbol{A} \boldsymbol{x}=\boldsymbol{b}$

where $\boldsymbol{A}$ is an $n \times n$ matrix, $\boldsymbol{x}$ and $\boldsymbol{b}$ are $n$-dimensional vectors. The elements of $\boldsymbol{A}, \boldsymbol{x}$, and $\boldsymbol{b}$ are denoted as $a_{i j}, x_{j}$, and $b_{j}(i, j=1$, $2, \cdots, n)$, respectively. Corresponding to Eq. (1), there always exists a function $f(\boldsymbol{x})$

$$
f(\boldsymbol{x})=\frac{1}{2} \boldsymbol{x}^{\mathrm{T}} \cdot \boldsymbol{A} \cdot \boldsymbol{x}-\boldsymbol{x}^{\mathrm{T}} \cdot \boldsymbol{b}
$$

where $\mathrm{T}$ means a transpose of a vector or a matrix. For a symmetric and positive definite matrix $\boldsymbol{A}$, the solution of Eq.(1) is equivalent to the vector which minimizes the function in Eq.(2). This leads directly to the conjugate gradient method ${ }^{[1-3,14]}$. If $\boldsymbol{A}$ is not symmetrical, a biconjugate gradient scheme ${ }^{[3]}$ is usually exploited instead.

The gradient of the function $f(\boldsymbol{x})$ in Eq.(2) is

$$
\nabla f=\boldsymbol{A} \cdot \boldsymbol{x}-\boldsymbol{b}
$$

provided that $\boldsymbol{A}$ is symmetrical. If $\nabla f$ is equal to zero, we obtain Eq. (1). This means that the solution of Eq. (1) corresponds to a stationary point of the function $f(\boldsymbol{x})$. The $j$ th component of the gradient is

$$
\frac{\partial f}{\partial x_{j}}=\sum_{k=1}^{n} a_{j k} x_{k}-b_{j}
$$

Similar to the derivation of correction vector in the Davidson's $\operatorname{method}^{[18]}$, if the element $x_{j}$ of the vector $\boldsymbol{x}$ is changed to $x_{j}+\delta_{j}$, keeping its other elements constant, Eq.(4) becomes

$$
\left.\frac{\partial f}{\partial x_{j}}\right|_{x_{j=x_{j}+\delta_{j}}}=\sum_{k=1}^{n} a_{j k} x_{k}-b_{j}+a_{i j} \delta_{j}
$$

If the right side of Eq. (5) is set equal to zero, we get a new vector $\boldsymbol{\delta}$ with its component $\delta_{j}$ defined as

$$
\delta_{j}=\left(b_{j}-\sum_{k=1}^{n} a_{j k} x_{k}\right) / a_{j j}
$$

The vector $\boldsymbol{\delta}$ is an improved vector for iterative solutions of Eq. (1). The numerator of the right side of Eq. (6) is actually a negative component of the gradient $\nabla f$, i.e., the steepest descent search direction. In the above derivation, it is assumed that the matrix $\boldsymbol{A}$ is symmetrical. However, numerical results as discussed below show that the vector $\boldsymbol{\delta}$ also works well for nonsymmetrical matrices.

The whole iterative scheme is described as follows. Start from a set of initial vectors $\left\{\boldsymbol{y}_{1}, \boldsymbol{y}_{2}, \cdots, \boldsymbol{y}_{l}\right\}, 1 \leq l<n$, they are assumed to be orthonormalized. The solution $\boldsymbol{x}$ of Eq. (1) can be expanded in this initial vector set as

$$
\boldsymbol{x}=\sum_{k=1}^{l} \alpha_{k} \boldsymbol{y}_{k}
$$

where $\alpha_{k}$ is the expansion coefficient to be determined. Substituting Eq.(7) into Eq.(1), after some manipulation, we get a new set of linear equations

$$
\boldsymbol{G} \boldsymbol{\alpha}=\boldsymbol{d}
$$

where the matrix elements of $\boldsymbol{G}$ and $\boldsymbol{d}$ are as follows

$$
g_{i j}=\boldsymbol{y}_{i}^{\mathrm{T}} \boldsymbol{A} \boldsymbol{y}_{j}, d_{i}=\boldsymbol{y}_{i}^{\mathrm{T}} \boldsymbol{b}(i, j=1,2, \cdots, l)
$$

The $k$ th component of the vector $\boldsymbol{\alpha}$ is the expansion coefficient $\alpha_{k}$ in Eq.(7). Since Eq.(8) is usually a small-scale linear equation, it can be solved simultaneously with conventional numerical methods such as Gaussian elimination method ${ }^{[1-3]}$. Once the expansion vector $\boldsymbol{\alpha}$ is available, we then calculate the residue $\boldsymbol{r}$ corresponding to the approximate vector $\boldsymbol{x}$

$$
\boldsymbol{r}=\boldsymbol{b}-\boldsymbol{A} \cdot \boldsymbol{x}
$$

If the norm $\|\boldsymbol{r}\|$ of $\boldsymbol{r}$ is less than some convergence criterion such as $10^{-8}$, the iterate process terminates. Otherwise, we get an improved vector $\boldsymbol{\delta}$ through Eq.(6). If $\boldsymbol{\delta}$ is not orthogonalized with the vectors $\left\{\boldsymbol{y}_{1}, \boldsymbol{y}_{2}, \cdots, \boldsymbol{y}_{l}\right\}$, some types of orothogonalization procedure such as the Schmidt method ${ }^{[22,23]}$ should be carried out. The orthonormal vector is denoted as $\boldsymbol{y}_{l+1}$. Then we go back to Eq.(7) with $(l+1)$ vectors and repeat the computations in Eq.(8) and Eq. (10) until the convergence threshold is reached.

\section{Numerical results}

For convenience of comparison with the conjugated gradient method and biconjugate gradient method, we start with one initial vector which can be chosen as a unit vector and then begin the iteration. Eq.(6) is always used to get an improved vector $\boldsymbol{\delta}$ even if matrix $\boldsymbol{A}$ is nonsymmetrical.

Four different examples are tested. The corresponding matrix elements of the first three examples are presented respectively below:

(a) $a_{i j}=\frac{1}{1+i+j}$

(b) $a_{i j}=a_{j i}=\left\{\begin{array}{ll}\frac{i}{r}+\frac{j}{s}, & i>j \\ i, & i=j\end{array}\right.$ with $r$ and $s$ as parameters;

(c) $a_{i j}=\left\{\begin{array}{l}\frac{i}{r}+\frac{j}{s}, \quad i \neq j \\ i, \quad, i=j\end{array}\right.$ with $r$ and $s$ as parameters.

Among them the first two matrices denoted as (a) and (b) are symmetrical. The matrices in the case (c) are nonsymmetrical. Before solving Eq. (1) the vector $\boldsymbol{b}$ is constructed by directly multiplying matrix $\boldsymbol{A}$ with the vector $\boldsymbol{x}$. Three types of the vector $\boldsymbol{x},\left\{x_{i}=0.1, i=1,2, \cdots, n\right\},\left\{x_{i}=0.5, i=1,2, \cdots, n\right\}$, and $\left\{x_{i}=\right.$ $i, i=1,2, \cdots, n\}$ are exploited. One purpose is to make sure that

\begin{tabular}{|c|c|c|c|c|}
\hline \multirow{2}{*}{ Size of matrix $\boldsymbol{A}$} & \multicolumn{2}{|c|}{ The present method } & \multicolumn{2}{|c|}{ CGM } \\
\hline & $10^{-6^{*}}$ & $10^{-8^{*}}$ & $10^{-6^{*}}$ & $10^{-8^{4}}$ \\
\hline \multicolumn{5}{|c|}{$x_{i}=0.5, i=1,2, \cdots, n$} \\
\hline 1000 & 7 & 7 & 184 & 208 \\
\hline 5000 & 7 & 8 & 419 & 472 \\
\hline \multicolumn{5}{|c|}{$x_{i}=i, i=1,2, \cdots, n$} \\
\hline 1000 & 7 & 9 & 204 & 227 \\
\hline 5000 & 9 & 9 & 461 & 515 \\
\hline
\end{tabular}
the program works correctly. Another one is to check the effect of the vector $\boldsymbol{b}$ on the convergence of the solution of Eq.(1).

The last example is the calculations of surface charges of the water cavity that nominally encloses the solute molecule $\mathrm{CH}_{3} \mathrm{CONH}_{2}{ }^{[13]}$. The cavity is a solute electronic isodensity contour with the isodensity value as 0.001 atomic unit. It is not

Table 1 Number of iterations for solving Eq.(1) in case (a) with the present method and CGM 
Table 2 Number of iterations for solving Eq.(1) in case (b) with the present method and CGM

\begin{tabular}{|c|c|c|c|c|c|}
\hline \multicolumn{2}{|c|}{ Parameter } & \multicolumn{2}{|c|}{ The present method } & \multicolumn{2}{|c|}{ CGM } \\
\hline$r^{*}$ & $s^{*}$ & $10^{-6^{* *}}$ & $10^{-8^{4 * 3}}$ & $10^{-6^{* * *}}$ & $10^{-0^{* * *}}$ \\
\hline \multicolumn{6}{|c|}{$n=1000,\left\{x_{i}=0.1, i=1,2, \cdots, n\right\}$} \\
\hline 10.0 & 20.0 & 13 & 15 & 161 & 195 \\
\hline 10.0 & 100.0 & 16 & 18 & 190 & 218 \\
\hline \multicolumn{6}{|c|}{$n=5000,\left\{x_{i}=0.1, i=1,2, \cdots, n\right\}$} \\
\hline 10.0 & 20.0 & 19 & 21 & 379 & 473 \\
\hline 10.0 & 100.0 & 23 & 25 & 461 & 546 \\
\hline \multicolumn{6}{|c|}{$n=1000,\left\{x_{i}=i, i=1,2, \cdots, n\right\}$} \\
\hline 10.0 & 20.0 & 17 & 19 & 219 & 245 \\
\hline 10.0 & 100.0 & 19 & 21 & 229 & 254 \\
\hline \multicolumn{6}{|c|}{$n=5000,\left\{x_{i}=i, i=1,2, \cdots, n\right\}$} \\
\hline 10.0 & 20.0 & 25 & 27 & 558 & 626 \\
\hline 10.0 & 100.0 & 29 & 31 & 607 & 669 \\
\hline
\end{tabular}

spherical. More details about this example and some related information can be found from Ref.[13] and references therein.

Quadruple precisions are used in all calculations below. The results for case (a) are listed in Table 1. The convergence criterion is $10^{-6}$ and $10^{-8}$. As can be seen from the table, it takes only 7-9 iterations for the present method to get a convergent solution. But it takes 184-515 iterations for the conjugate gradient method to obtain the same results. Another advantage of the present method is that the number of iterates remains almost unchanged when the dimension of matrix $\boldsymbol{A}$ increases from 1000 to 5000 . However, the number of iterates required for CGM is more than doubled.

The results for case (b) are presented in Table 2. In comparison with CGM, the advantage of the present method is still very significant though the differences are not as large as those in case (a). The next example is the nonsymmetrical case (c). The results are listed Table 3. The differences between the present method and BCGM are very large. The present method needs only 3 or 4 iterations to get convergent solutions while BCGM requires at

Table 3 Number of iterations for solving Eq.(1) in case (c) with the present method and BCGM

\begin{tabular}{|c|c|c|c|c|c|}
\hline \multicolumn{2}{|c|}{ Parameter } & \multicolumn{2}{|c|}{ The present method } & \multicolumn{2}{|c|}{ BCGM } \\
\hline$r^{*}$ & $s^{*}$ & $10^{-6^{* *}}$ & $10^{-8^{8 *}}$ & $10^{-6^{* *}}$ & $10^{-8^{4}}$ \\
\hline \multicolumn{6}{|c|}{$n=1000,\left\{x_{i}=0.1, i=1,2, \cdots, n\right\}$} \\
\hline 10.0 & 20.0 & 3 & 3 & 170 & 201 \\
\hline 10.0 & 100.0 & 3 & 3 & 181 & 210 \\
\hline \multicolumn{6}{|c|}{$n=5000,\left\{x_{i}=0.1, i=1,2, \cdots, n\right\}$} \\
\hline 10.0 & 20.0 & 3 & 3 & 388 & 465 \\
\hline 10.0 & 100.0 & 3 & 3 & 409 & 480 \\
\hline \multicolumn{6}{|c|}{$n=1000,\left\{x_{i}=i, i=1,2, \cdots, n\right\}$} \\
\hline 10.0 & 20.0 & 4 & 4 & 222 & 245 \\
\hline 10.0 & 100.0 & 4 & 4 & 218 & 242 \\
\hline \multicolumn{6}{|c|}{$n=5000,\left\{x_{i}=i, i=1,2, \cdots, n\right\}$} \\
\hline 10.0 & 20.0 & 4 & 4 & 532 & 589 \\
\hline 10.0 & 100.0 & 4 & 4 & 526 & 580 \\
\hline
\end{tabular}

Table 4 Number of iterations for solving Eq.(1) for the cavity surface charges of $\mathrm{CH}_{3} \mathrm{CONH}_{2}$ solvent with the present method and BCGM

\begin{tabular}{|c|c|c|c|c|}
\hline \multirow{2}{*}{ Size of matrix $\boldsymbol{A}$} & \multicolumn{2}{|c|}{ The present method } & \multicolumn{2}{|c|}{ BCGM } \\
\hline & $10^{-6^{*}}$ & $10^{-8^{*}}$ & $10^{-6^{*}}$ & $10^{-8^{*}}$ \\
\hline 4002 & 30 & 40 & 35 & 58 \\
\hline 5762 & 31 & 43 & 37 & 63 \\
\hline
\end{tabular}

least 170 iterations. The maximum number of iterations for BCGM is 589 with the dimension of matrix $\boldsymbol{A}$ as 5000 .

It is also found from the results in Tables 1,2 , and 3 that the vector $\boldsymbol{b}$ and the convergence threshold have almost no effect on the speed of convergence of the present method in terms of iterations, but do have some influence on the convergence of CGM and BCGM though the extents are not as large as the size of matrix $\boldsymbol{A}$.

The surface charges of the water cavity that nominally encloses the solute molecule $\mathrm{CH}_{3} \mathrm{CONH}_{2}$ have been calculated with the so-called SS(V)PE (surface and simulation of volume polarization for electrostatics) method ${ }^{[13,24,25]}$ implemented in $\mathrm{HONDO}^{[2]}$. The algebraic form of SS(V)PE method is a set of linear equations. The corresponding matrix is nonsymmetrical and diagonally dominant. The linear equations are solved with the present method and BCGM, respectively. The numerical results are listed in Table 4. The sizes of the matrix $\boldsymbol{A}$ are 4002 and 5762, respectively. It can be seen from Table 4 that the performances of the present method are also better than BCGM.

Some other types of well-conditioned matrices, for example, the configuration interaction matrices with dimensions larger than 100000 , have also been investigated. The numerical results show that the present method is still superior to CGM.

\section{Conclusions}

The Davidson's method was extended to solve of large-scale linear equations. Numerical results in this study demonstrate that the present scheme is superior to the conjugate gradient method and the biconjugate gradient method for both symmetrical and nonsymmetrical cases, respectively. It seems that the present scheme is especially efficient for the nonsymmetrical and nondiagonal dominant matrices.

\section{References}

1 Quarteroni, A.; Sacco, R.; Saleri, F. Numerical mathematics. New York: Springer-Verlag, 2007

2 Burden, R. L.; Fairs, J. D. Numerical analysis. 7th ed. Pacific Grove: Brooks/Cole Publishing, 2001

3 Press, W. H.; Teukolsky, S. A.; Vetterling, W. T.; Flannery, B. P. Numerical recipes in Fortran. 2nd ed. New York: Cambridge University Press, 1986

4 Ye, H. X.; Jin, Y. Q. Acta Phys. Sin., 2008, 57: 839 [叶红霞, 金 亚秋. 物理学报, 2008, 57: 839]

5 Cao, Y. L.; Wang, X. S. Acta Phys. -Chim. Sin., 2004, 20: 785 
[曹益林, 汪彭松. 物理化学学报, 2004, 20: 785]

6 Wu, X. P.; Wang, T. T. Acta Geophys. Sin., 2003, 46: 428 [吴小平, 汪肜朋地球物理学报, 2003, 46: 428]

7 Xiong, F. L.; Guan, Z. N. Acta Geophys. Sin., 1992, 35: 249 [熊富林, 管志宁. 地球物理学报, 1992, 35: 249]

8 Li, J. K.; Jiang, L. Y.; Liu, Y. Acta Geophys. Sin., 1994, 37: 616 [李家康, 姜兰茵, 刘 宇. 地球物理学报, 1994, 37: 616]

9 Pople, J. A.; Krishnan, R.; Schlegel, H. B.; Binkley, J. S. Int. J. Quantum Chem. Symp., 1979, 13: 225

10 Pervis III, G. D.; Bartlett, R. J. J. Chem. Phys., 1981, 75: 1284

11 Ramasesha, S. J. Comput. Chem., 1990, 11: 545

12 Shuai, Z.; Ramasesha, S.; Brédas, J. L. Chem. Phys. Lett., 1996, 250: 14

13 Chen, F.; Chipman, D. M. J. Chem. Phys., 2003, 119: 10289

14 Watowich, S. J.; Meyer, E. S.; Hagstrom, R.; Josephs, R. J. Comput. Chem., 1988, 9: 650

15 Morgan, R. B. SIAM J. Sci. Comput., 2002, 24: 20

16 Paige, C. C.; Strakoš, Z. SIAM J. Sci. Comput., 2002, 23: 1898
17 Lötstedt, P.; Nilsson, M. SIAM J. Sci. Comput., 2004, 25: 2126

18 Davidson, E. R. J. Comput. Phys., 1975, 17: 87

19 Murray, C. W.; Racine, S. C.; Davidson, E. R. J. Comput. Phys., 1992, 103: 382

20 Lenthe, J. H.; Pulay, P. J. Comput. Chem., 1990, 11: 1164

21 Zhao, X. H.; Chen, F. W.; Wu, J.; Zhao, Q. L. Acta Phys. -Chim. Sin., 2008, 24: 823 [赵小红, 陈飞武, 吴 健, 周巧龙. 物理化 学学报, 2008, 24: 823]

22 Chen, F. W. Computational methods in quantum chemistry. Beijing: Science Press, 2008: 166-170 [陈飞武. 量子化学中的 计算方法. 北京: 科学出版社, 2008: 166-170]

23 Szabo, A.; Ostlund, N. S. Modern quantum chemistry: introduction to advanced electronic structure theory. New York: Macmillan, 1982

24 Chipman, D. M. J. Chem. Phys., 1999, 110: 8012

25 Chipman, D. M. J. Chem. Phys., 2000, 112: 5558

26 Dupuis, M.; Marquez, A.; Davidson, E. R. HONDO 2001, based on HONDO-95. 1977 